\title{
Transverse to longitudinal phase space coupling in an electron beam for suppression of microbunching instability
}

\author{
Dazhang Huang, Chao Feng, 'Haixiao Deng, Qiang Gu, and Zhentang Zhao \\ Shanghai Institute of Applied Physics, Chinese Academy of Sciences, Shanghai 201800, China
}

(Received 26 April 2016; published 3 October 2016)

\begin{abstract}
The microbunching instability developed during the beam compression process in the linear accelerator (LINAC) of a free-electron laser (FEL) facility has always been a problem that degrades the lasing performance, and even no FEL is able to be produced if the beam quality is destroyed too much by the instability. A common way to suppress the microbunching instability is to introduce extra uncorrelated energy spread by the laser heater that heats the beam through the interaction between the electron and laser beam, as what has been successfully implemented in the Linac Coherent Light Source and Fermi@Elettra. In this paper, a simple and effective scheme is proposed to suppress the microbunching instability by adding two transverse gradient undulators (TGU) before and after the magnetic bunch compressor. The additional uncorrelated energy spread and the density mixing from the transverse spread brought up by the first TGU results in significant suppression of the instability. Meanwhile, the extra slice energy spread and the transverse emittance can also be effectively recovered by the second TGU. The magnitude of the suppression can be easily controlled by varying the strength of the magnetic fields of the TGUs. Theoretical analysis and numerical simulations demonstrate the capability of the proposed technique in the LINAC of an x-ray free-electron laser facility.
\end{abstract}

DOI: 10.1103/PhysRevAccelBeams.19.100701

\section{INTRODUCTION}

$\mathrm{X}$-ray free-electron lasers (FELs) hold great promise as ultrashort, tunable, intensity radiation sources for advanced user applications and open up new frontiers of ultrafast and ultrasmall sciences at the atomic scale. In recent years, the successful user operation of the first FEL facilities [1-4] in soft and hard x-ray regimes announced the birth of the x-ray free-electron laser. In the x-ray FEL process, the required high intensity electron beams of subpicosecond (sub-ps) length are usually obtained by compressing longer beams in magnetic bunch compressors at relativistic energies. The bunch compressor manipulates longitudinal phase space of the electron beam with a considerable energy chirp by introducing the dependence of a particle's longitudinal position on its energy. As a result, the bunch can be significantly compressed in the bunch compressor due to the momentum compaction factor $\left(R_{56}\right)$ therein. On the other hand, due to existence of the longitudinal space charge (LSC), the coherent synchrotron radiation (CSR) and structural impedance in the beam transportation and compression process, the initial small energy and density perturbation in the electron bunch can be amplified with a large gain factor in many cases, which results in the

\footnotetext{
fengchao@sinap.ac.cn

Published by the American Physical Society under the terms of the Creative Commons Attribution 3.0 License. Further distribution of this work must maintain attribution to the author $(s)$ and the published article's title, journal citation, and DOI.
}

increase of the fragmentation of the longitudinal phase space and dilute the emittance [5-9]. This process of amplification is usually known as the microbunching instability, and it will seriously degrade the FEL performance thereafter.

In recent decades, many techniques that rely on electron beam manipulation [10-14] have been developed to suppress the microbunching instability. The most popular and reliable technique used in a number of facilities is a laser heater [15-17], which employs a polarized laser pulse in a short undulator located in the middle of a chicane to increase the uncorrelated energy spread of the electron beam [12] through the interaction between electron and laser beam. Because the electron beam emitted from a high brilliance photocathode gun usually has an extremely small slice energy spread on the order of a few $\mathrm{keV}[17,18]$, the laser heater is developed to provide the additional slice energy spread to an extent that reduces the gain of the instability via Landau damping. By carefully choosing the power of the laser pulse, the laser-induced slice energy spread could be adjusted precisely to optimize the FEL working point. However, although this laser-induced energy spread is tolerable for self-amplified spontaneous emission FELs, it limits the effectiveness of seeding schemes and may also introduce the side effects such as trickle heating [15]. Recently, alternative techniques $[10,11,13,14]$ have also been proposed for suppressing the microbunching instability based on other methods of electron beam manipulation, such as deflecting cavity [10] and bending magnets [11], etc., but many of the newly 
developed methods are relatively complex and not as easy to be applied to the existing FEL facilities.

The original idea of using a transverse gradient undulator (TGU) to mitigate the effects of electron beam energy spread in FEL oscillators was proposed in Ref. [19]. It was found later that the TGU is a functional device that provides an additional measure for manipulating the electron beam via transverse-to-longitudinal phase space coupling. One of the applications of this manipulation technique is to perform the phase merging effect for significantly improving the frequency up-conversion efficiency of a seeded FEL $[20,21]$. In this paper, by investigating the transport matrix and the beam dynamics in a TGU, a simple and inexpensive technique based on transverse-to-longitudinal phase space coupling is proposed to suppress the microbunching instability of an electron beam. It is found that by adding a TGU right before the magnetic bunch compressor where large energy chirp exists, the gain of the microbunching instability developed in the electron beam can be effectively suppressed by the additional slice energy spread and the density mixing from the transverse spread introduced by the TGU. Meanwhile, the additional slice energy spread and the transverse emittance growth introduced by the first TGU can also be recovered very well by another TGU right after the compressor. Compared with the other techniques, this method is quite simple and could be easily applied to all existing FEL facilities in addition to a laser heater.

Previously, we have investigated the similar scheme to suppress the microbunching instability by only one TGU before compression [22]. The result indicates that the growth of the instability can be effectively suppressed. The prerequisite of this method is the very small initial global beam energy spread before bunch compression for the preservation of the transverse emittance, which needs the special techniques such as the back-tracking method [23-25]. However, the new scheme proposed in this article is applicable for a general beam without any special requirement. Moreover, in this new scheme, the extra beam slice energy spread can also be introduced by the first TGU through transverse-longitudinal coupling before compression, which is very helpful for the suppression of the microbunching instability and can be considered as another advantage over the schemes in Refs. [11,22].

In Sec. II, the methods to suppress the microbunching instability by TGUs are investigated analytically and the equations are derived to explain the whole idea. In Sec. III, the numerical simulation is carried out to demonstrate the idea based on an example lattice. The jitter issues that may appear in a real machine are discussed in Sec. IV, and the conclusion remarks are made in Sec. V.

\section{METHODS}

A transverse gradient undulator (TGU) is an undulator with a transverse gradient between the magnetic poles. The original idea of the TGU was a tool to prevent gain degradation in FEL oscillators due to the large energy spread of the electron beam [19]. Recently, the idea has been applied to laser-plasma accelerator-driven high-gain FELs $[26,27]$ for the design of compact X-ray FEL devices. The TGU can be realized by canting the poles of a regular undulator and the gradient is usually made in the horizontal direction. Because the electrons at different horizontal positions feel different magnetic fields, the path length of an electron traversing a TGU depends on its transverse coordinates at the entrance of the TGU; as a result, the transverse-to-longitudinal phase space coupling is introduced. Ignoring the vertical effects, the first-order transport matrix of the TGU in $\left(\vec{x}, x^{\prime}, \vec{z}, \delta_{\gamma}\right)$ phase space can be derived as

$$
\mathbf{R}_{\mathbf{T G U}}=\left[\begin{array}{cccc}
1 & L_{T} & 0 & \tau L_{T} / 2 \\
0 & 1 & 0 & -\tau \\
\tau & \tau L_{T} / 2 & 1 & -\tau^{2} L_{T} / 6 \\
0 & 0 & 0 & 1
\end{array}\right],
$$

where $L_{T}$ is the effective length of TGU and $\tau$ is the strength of TGU. In the TGU transport matrix (1), one can see that the effective elements $R_{31}$ and $R_{24}$ are of the same value but in opposite signs.

Assuming a TGU is placed right before the first dipole of a magnetic bunch compressor where the energy chirp is large, because of the transverse-to-longitudinal coupling introduced by the TGU, it turns out that the electrons at the different transverse locations in the bunch have different path lengths traversing the TGU, which results in the redistribution of the longitudinal beam phase space and increases the longitudinally slice energy spread, or called heating. In the following, we start our investigation for the beam with linear energy chirp. To study the behavior of the microbunching instability in the presence of the TGUs in a reasonable way, the density perturbation in one wavelength is divided into multiple slices. Because the wavelength of the microbunching instability is usually much shorter than the bunch length, the assumption of the uniform longitudinal density distribution within a beam slice is employed in the following discussion.

\section{A. Analytical solution}

Previously, we have already discussed the physical mechanism of the TGU for suppression of the microbunching instability with reasonable approximations [22]. In the following, we will be discussing the mechanism for the general case. If a linear energy chirp is added on the electron beam with Gaussian energy distribution before entering into the TGU, the longitudinal phase space distribution of the beam particles reads [5]

$$
f_{0}\left(z, \delta_{\gamma}\right)=\frac{I_{0}}{\sqrt{2 \pi} \sigma_{\gamma}} \exp \left[-\frac{\left(\delta_{\gamma}-h \gamma_{0} z\right)^{2}}{2 \sigma_{\gamma}^{2}}\right] .
$$


Here we define $z$ the longitudinal coordinate of a beam particle within the bunch, where $z=0$ represents the beam center, and $z>0$ is behind the beam center. $I_{0}$ is the longitudinal beam current, $\gamma_{0}$ is the relativistic central beam energy, $\sigma_{\gamma}$ is the initial slice energy spread, $\delta_{\gamma}=\delta \gamma\left(\gamma_{0}\right)^{-1}$ is the energy deviation of a particle, where $\gamma$ represents the energy of the particle and $h=d \delta \gamma(\gamma d z)^{-1}$ is used for quantifying the beam energy chirp. After passing through the short TGU with period length $\lambda_{u}$, period number $N_{u}$, transverse gradient $\alpha$ and central undulator parameter $K_{0}$, electrons with different horizontal positions $x$ will see different $K$ values, where $K(x)=K_{0}(1+\alpha x)$ [18]. According to our discussions above and based on the TGU matrix (1), it results in different path lengths and converts the longitudinal coordinate into

$$
z_{f}=z+\tau x+\frac{\tau L_{T}}{2} x^{\prime}-\frac{\tau^{2} L_{T}}{6} \delta_{\gamma}
$$

where $L_{T}=N_{u} \lambda_{u}$ represents the length of TGU and $\tau=L_{T} K_{0}^{2} \alpha\left(2 \gamma^{2}\right)^{-1}$ is the gradient parameter of TGU for particle energy $\gamma$.

Without losing generality, a beam of Gaussian distributions in $x$ and $x^{\prime}$ without correlation was employed in our study. After traversing the TGU, the distribution of the beam particles within a longitudinal thin slice can be obtained by substituting the longitudinal coordinate $z$ in Eq. (2) by $z_{f}$ :

$$
\begin{aligned}
f_{0}\left(z, x, \delta_{\gamma}\right)= & \frac{I_{0}}{(2 \pi)^{3 / 2} \sigma_{\gamma} \sigma_{x} \sigma_{x^{\prime}}} \\
& \times \exp \left(-\frac{x^{2}}{2 \sigma_{x}^{2}}\right) \exp \left(-\frac{x^{\prime 2}}{2 \sigma_{x^{\prime}}^{2}}\right) \\
& \times \exp \left[-\frac{\left(\tilde{\delta}_{\gamma}-h \gamma_{0} z-h \gamma_{0} \tau x-h \gamma_{0} \tau L_{T} x^{\prime} / 2\right)^{2}}{2 \sigma_{\gamma}^{2}}\right],
\end{aligned}
$$

where $x$ is the horizontal position of a beam particle and $x=0$ is in the center of the beam. To simplify the equation, $\tilde{\delta}_{\gamma}=\delta_{\gamma}\left(1+h \gamma_{0} \tau^{2} L_{T} / 6\right)$ is defined. For a sufficiently thin beam slice, we make the assumption that all the particles within the slice have the same longitudinal coordinate $z$. It is found in Eq. (4) that the horizontally correlated energy spread is converted into longitudinal slice energy spread with the energy chirp $h \tau$, which increases the slice energy spread of the beam before compression. As a result, the gain of the microbunching instability during compression is reduced. Without losing generality, we look at the central part of the beam where the slice energy spread is almost the same all along. To simply the problem, for the middle slice where $z=0$, and given the horizontal beam size $\sigma_{x}$, we do the integral along the horizontal axis and obtain the energy distribution without horizontal dependency,

$$
\begin{aligned}
f_{0}\left(\tilde{\delta}_{\gamma}\right)= & \int_{-\infty}^{\infty} d x \int_{-\infty}^{\infty} d x^{\prime} f_{0}\left(z=0, x, \tilde{\delta}_{\gamma}\right) \\
= & \frac{I_{0}}{2 \pi \sigma_{\gamma} \sigma_{x} \sigma_{x^{\prime}}} \frac{\sigma_{\gamma} \sigma_{x} \sigma_{x^{\prime}}}{2} \\
& \times \sqrt{\frac{2 \pi}{\sigma_{\gamma}^{2}+\left(h \tau \gamma_{0} \sigma_{x}\right)^{2}+\left(h \gamma_{0} \sigma_{x^{\prime}} \tau L_{T} / 2\right)^{2}}} \\
& \times \exp \left[-\frac{\tilde{\delta}_{\gamma}^{2}}{2\left[\sigma_{\gamma}^{2}+\left(h \tau \gamma_{0} \sigma_{x}\right)^{2}+\left(h \gamma_{0} \sigma_{x^{\prime}} \tau L_{T} / 2\right)^{2}\right]}\right] \\
= & \times \operatorname{erf}(X) \mid I_{x=-\infty}^{x=\infty} \\
& \sqrt{2 \pi\left[\sigma_{\gamma}^{2}+\left(h \tau \gamma_{0} \sigma_{x}\right)^{2}+\left(h \gamma_{0} \sigma_{x^{\prime}} \tau L_{T} / 2\right)^{2}\right]} \\
& \times \exp \left[-\frac{\tilde{\delta}_{\gamma}^{2}}{2\left[\sigma_{\gamma}^{2}+\left(h \tau \gamma_{0} \sigma_{x}\right)^{2}+\left(h \gamma_{0} \sigma_{x^{\prime}} \tau L_{T} / 2\right)^{2}\right]}\right],
\end{aligned}
$$

where $X$ is a linear function of $x$ and $\operatorname{erf}(X)=$ $2 \pi^{-0.5} \int_{0}^{X} e^{-t^{2}} d t$ is the error function.

Equation (5) shows us a typical Gaussian distribution function with the rms energy spread

$$
\sigma_{\gamma}^{\prime}=\sqrt{\sigma_{\gamma}^{2}+\left(h \tau \gamma_{0} \sigma_{x}\right)^{2}+\left(h \gamma_{0} \sigma_{x^{\prime}} \tau L_{T} / 2\right)^{2}},
$$

which has the contributions from position divergence $\sigma_{x}$, angular divergence $\sigma_{x^{\prime}}$, gradient parameter $\tau$ and effective length $L_{T}$ of the TGU. This new energy spread can be much larger than the original one $\sigma_{\gamma}$. Again, because the slice energy spread in the vicinity of the beam center does not vary much, the energy distribution of the beam at slice $z$ around the center of a chirped beam traversing a TGU can be finally written as

$$
f_{0}\left(z, \delta_{\gamma}\right)=\frac{I_{0}}{\sqrt{2 \pi} \sigma_{\gamma}^{\prime}} \exp \left[-\frac{\left(\delta_{\gamma}-h \gamma_{0} z\right)^{2}}{2 \sigma_{\gamma}^{\prime 2}}\right] .
$$

On the other hand, the TGU is also able to introduce the longitudinal mixing from the transverse spread, which is considered as another big advantage to suppress the microbunching instability. Following the similar methods in Refs. [5,9,11], and making use of the current distribution function in [5], which is

$$
I(s)=\frac{I_{0}}{\sqrt{2 \pi} \sigma_{\gamma}^{\prime}} \int_{-\infty}^{\infty} d \delta \gamma \exp \left[-\frac{\left[\delta \gamma\left(1+h R_{56}\right)-h \gamma_{0} z+\Delta \gamma \sin \left(k z-k R_{56} \delta \gamma / \gamma_{0}\right)\right]^{2}}{2 \sigma_{\gamma^{\prime}}^{2}}\right],
$$


where $R_{56}$ is the momentum compaction of the bunch compressor, $k=k_{0}\left(1+h R_{56}\right)$ is the modulation wave number after compression, with $k_{0}$ the modulation wave number before compression and $h$ the energy chirp. $\Delta \gamma$ represents the energy modulation amplitude defined in [5] and $\sigma_{\gamma}^{\prime}$ is the equivalent energy spread defined in Eq. (6). By substituting $z_{f}$ in Eq. (3) into Eq. (8) as $z$, in such a way to take into account the TGU effects, and following the same procedure in [5], we obtain the final gain of the microbunching instability after the passage through the bunch compressor with TGU taking effects

$$
\begin{aligned}
G_{f}= & G_{0} \exp \left(-\frac{k^{2} R_{56}^{2} \sigma_{\gamma}^{\prime 2}}{2 \gamma_{0}^{2}}\right) \exp \left(-\frac{k^{2} \tau^{2} \sigma_{x}^{2}}{2}\right) \\
& \times \exp \left(-\frac{k^{2} \tau^{2} L_{T}^{2} \sigma_{x^{\prime}}^{2}}{8}\right) \exp \left(-\frac{k^{2} \tau^{4} L_{T}^{2} \sigma_{\gamma^{\prime}}^{2}}{72 \gamma_{0}^{2}}\right) \\
\approx & G_{0} \exp \left(-\frac{k^{2} R_{56}^{2} \sigma_{\gamma}^{\prime 2}}{2 \gamma_{0}^{2}}\right) \exp \left[-\left(\frac{k^{2} \tau^{2} \sigma_{x}^{2}}{2}+\frac{k^{2} \tau^{2} L_{T}^{2} \sigma_{x^{\prime}}^{2}}{8}\right)\right],
\end{aligned}
$$

where $G_{0}=k\left|R_{56}\right| I_{0} Z(k)\left(\gamma_{0} I_{A} Z_{0}\right)^{-1}$, with $Z_{0}=377 \Omega$ the free-space impedance and $Z(k)$ the longitudinal impedance of wave number $k$. Note that the last term with $\tau^{4}$ is dropped because the independent variable inside the exponential function is much smaller than the first three.

In Eq. (9), the terms on the right-hand side provide us the way for suppressing the microbunching instability. In the equation, one can see that the gain of the microbunching instability can be damped by the two factors introduced by the transverse-to-longitudinal coupling throughout the TGU: one is the additional slice energy spread in the presence of significant beam energy chirp, another is the smearing of the microbunches caused by the longitudinal mixing from the transverse spread.

The discussions above enlighten us to implement the TGUs into the LINAC for the suppression of the microbunching instability. The layout of the proposed lattice is shown in Fig. 1. In the lattice, two TGUs with the opposite gradient parameters are symmetrically placed on both sides of the magnetic bunch compressor.

\section{B. Emittance preservation and cancellation of additional uncorrelated energy spread}

Based on the discussions above, the TGUs introduce additional dispersion into the lattice. It is a standard way to make the lattice dispersion-free by employing one or more

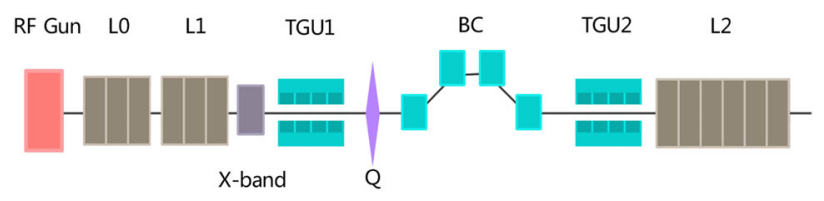

FIG. 1. Lattice layout of TGU suppression scheme. quadrupoles to eliminate the additional dispersion introduced by the TGUs. Indeed, more quadruples give more flexibility for the lattice optimization. In our example, to simplify the problem, only one quadruple is implemented in between TGU1 and the bunch compressor (BC), as illustrated in Fig. 1. In the following discussion, it will show that the solutions can be found to make the whole lattice dispersion-free in such a way to preserve the transverse emittance.

In the first-order transport matrix of the TGU [see in (1)], the matrix elements $R_{14}$ and $R_{24}$ introduce additional horizontal emittance growth because of the coupling. To avoid such an emittance growth, by investigating the first-order transport matrix from TGU1 to TGU2 in Fig. 1 and assuming that the quadrupole is of the same distance to TGU1 and to the first dipole of the compressor, we have

$$
\mathbf{M}=\mathbf{R}_{\mathrm{TGU} 2} \cdot \mathbf{R}_{\mathrm{d} 3} \cdot \mathbf{R}_{\mathrm{BC}} \cdot \mathbf{R}_{\mathrm{d} 2} \cdot \mathbf{R}_{\mathbf{Q}} \cdot \mathbf{R}_{\mathrm{d} 1} \cdot \mathbf{R}_{\mathrm{TGU} 1},
$$

where $\mathbf{d} \mathbf{1}, \mathbf{d} \mathbf{2}$ and $\mathbf{d} \mathbf{3}$ represent the drift spaces before and after the quadrupole, and that between the compressor and TGU2, respectively. To simplify the problem, let $\mathbf{d} \mathbf{1}=\mathbf{d} 2=L_{d}, \mathbf{d} \mathbf{3}=2 L_{d}, L_{T 1}=L_{T 2}=L_{T}$ and $\tau_{1}=-\tau_{2}=\tau$. By applying the thin lens approximation, finally the coupling elements take the form

$$
\begin{aligned}
M_{14}= & -\tau\left(4 L_{B}+2 L_{12}+L_{23}+4 L_{d}+L_{q}+L_{T}\right) \\
& +\frac{1}{2} \tau \tilde{k}^{2} L_{q}\left(2 L_{d}-L_{T}\right)\left(4 L_{B}+2 L_{12}\right. \\
& \left.+L_{23}+3 L_{d}+L_{T}\right)
\end{aligned}
$$

and

$$
M_{24}=\frac{1}{2} \tau \tilde{k}^{2} L_{q}\left(2 L_{d}-L_{T}\right)
$$

where $\tilde{k}$ is the quadrupole strength and $\tilde{k}^{2}=$ $\left(d B_{y} / d x\right)\left(1 / B_{0} \rho_{0}\right)$ with $B_{0} \rho_{0}$ the magnetic rigidity of the central trajectory, and $d B_{y} / d x$ the horizontal gradient of the vertical magnetic field component in the quadrupole [28], $L_{12}=L_{34}$ is the distance between the first and the last two dipole magnets in the bunch compressor, and $L_{23}$ is the distance between the two dipoles in the middle of the compressor, $L_{B}$ is the effective length of the dipole (bending) magnet and $L_{q}$ is the effective length of the quadrupole.

To eliminate the additional transverse emittance growth, it needs $M_{14}=0$ and $M_{24}=0$. For matrix element $M_{24}$, one can easily tell that with $\tau \ll 1$ and the thin lens approximation, $M_{24} \rightarrow 0$. For matrix element $M_{14}$, one can have it equal to 0 by tuning the quadrupole strength $k$ to make 


$$
\tilde{k}^{2}=\frac{2\left(4 L_{B}+2 L_{12}+L_{23}+4 L_{d}+L_{q}+L_{T}\right)}{L_{q}\left(2 L_{d}-L_{T}\right)\left(4 L_{B}+2 L_{12}+L_{23}+3 L_{d}+L_{T}\right)} .
$$

Given the regular length of the TGU, the expression on the right-hand side of Eq. (13) will always be larger than zero, therefore Eq. (13) will always have real solutions of $\tilde{k}$. Based on the fact that $M_{24} \rightarrow 0$ and Eq. (13), the whole suppression lattice will be dispersion-free and no extra transverse emittance growth will be introduced.

On the other hand, in Sec. II A we have derived the explicit formula of the uncorrelated slice energy spread of a chirped beam after traversing a TGU [Eq. (6)], with the extra slice energy spread introduced by the TGU included. If we let the beam pass through the whole lattice represented by matrix (10), with the thin lens approximation and the fact that $L_{T}$ is usually much smaller than the total length of the bunch compressor and $L_{d}$, we have the matrix elements $M_{31}=0$ and $M_{32}=0$ by applying Eq. (13). Therefore we conclude that the additional slice energy spread introduced by TGU1 through the transverse-longitudinal coupling will also be recovered at the exit of TGU2 in our scheme. The explicit form of matrix $\mathbf{M}$ is shown in (14), where $L_{\text {tot }}$ is the total length from TGU1 to TGU2, and $M_{34}$ is equivalent to $R_{56}$ in the six-dimensional transport matrix, which will be discussed in more detail in Sec. IV.

$$
\mathbf{M}=\left[\begin{array}{cccc}
1 & L_{\mathrm{tot}} & 0 & 0 \\
0 & 1 & 0 & 0 \\
0 & 0 & 1 & M_{34} \\
0 & 0 & 0 & 1
\end{array}\right]
$$

In the next section, we will present a proof of principle of this scheme by numerical simulation and one can clearly see a significant decrease of the energy and current fluctuations at the end of the LINAC with the TGUs implemented in the lattice.

\section{NUMERICAL RESULTS}

In this section, we will show the proof of principle and possible performance of the proposed double-TGU technique for the suppression of microbunching instability illustrated in Fig. 1. As usual, an X-band radio-frequency (rf) structure is employed before the first TGU to compensate the second-order nonlinear components in the longitudinal phase space to avoid the undesired growth of transverse emittance and energy spread. For simplicity purposes, we just look at one compression stage. L1 and L2 are two accelerating sections to provide and compensate the energy chirp to the beam before and after the bunch compressor. Without losing generality, the nominal beam and LINAC parameters [29] were employed in the simulation and are shown in Table I. Moreover, since the length
TABLE I. Nominal beam and linac parameters in simulation.

\begin{tabular}{lc}
\hline \hline Parameter & Value \\
\hline Bunch charge (nC) & 1.0 \\
Beam energy before BC (MeV) & 245 \\
Bunch length (FWHM) before BC (ps) & 7 \\
Peak beam current before BC (A) & 110 \\
Slice beam energy spread (rms) before BC (keV) & 3 \\
Linac length up to BC (m) & 17.3 \\
$R_{56}$ of BC (mm) & -72 \\
Beam compression ratio & $\sim 9$ \\
\hline \hline
\end{tabular}

scale in which the structural impedance is effective is much longer than that of microbunching wavelength [30,31], we may neglect the effects from the linac wakefields in the simulation without compromising accuracy.

In order to emulate a general case, the Gaussian beam in the six-dimensional phase space is employed, and the density modulation is added on the beam profile before entering into TGU1 (Fig. 2) with peak-to-peak amplitude of $10 \%$, and $50 \mu \mathrm{m}$ in wavelength. The particle tracking code ELEGANT [32] is used to do the simulation in linac and a $3 \mathrm{D}$ algorithm based on the fundamentals of electrodynamics [33] is employed to do the simulation in TGU. The simulation starts before TGU1 where the beam energy is about $245 \mathrm{MeV}$, the peak current is about $110 \mathrm{~A}$, and with the relative energy chirp $\sim 0.025 \mathrm{~mm}^{-1}$. As mentioned above, a variable-gap TGU (TGU1) with ten periods of $10 \mathrm{~cm}$ period length, $B_{0} \approx 2.0 \mathrm{~T}$ and transverse gradient $\alpha=66 \mathrm{~m}^{-1}$ is adopted before compression. The electron beam is then compressed in the ratio of 9 in the magnetic bunch compressor. After that, another TGU (TGU2) with the same parameters as TGU1 except the opposite direction of the transverse gradient is used to recover the transverse emittance and the energy spread. Initially, the horizontal and the vertical beam size $\sigma_{x, y} \approx 0.4 \mathrm{~mm}$ with the angular spread $\sigma_{x^{\prime}, y^{\prime}} \approx 0.035 \mathrm{mrad}$ at the entrance of TGU1. Ten million macroparticles with total charge of $1.0 \mathrm{nC}$ are used in the simulation.

To save computing time, the simulation starts right before TGU1 and ends after TGU2 (Fig. 1) instead of the linac exit. This makes no difference because the

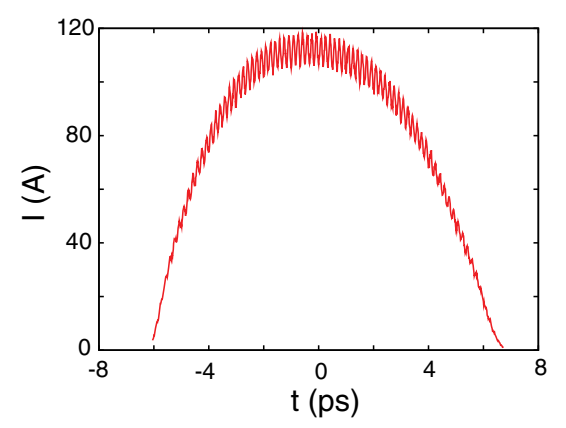

FIG. 2. Initial longitudinal beam current profile. 


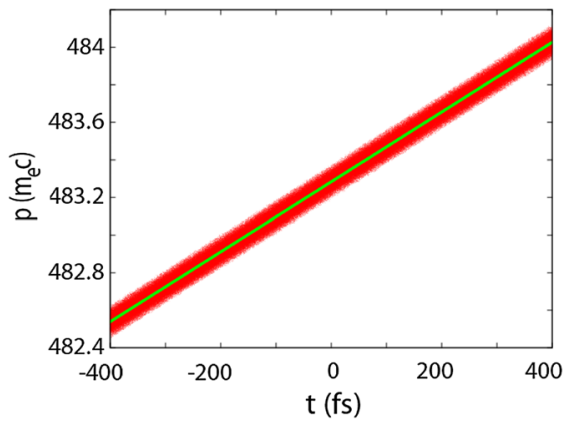

FIG. 3. Longitudinal phase space (time momentum) of the central part of the beam before (green) and after (red) TGU1.

microbunching instability information at these locations is adequate to demonstrate the problem, and the remnant correlated energy chirp at these locations plays no role in our discussion. For comparison, Fig. 3 shows the phase space of the central part of the beam before and after TGU1. In the figure, one can clearly see the significant growth of the slice energy spread done by TGU1. Figure 4 shows the longitudinal beam current and the slice energy profile of the central part of the beam with the TGUs on and off, in which one can clearly see that the microstructures introduced by the microbunching instability during the compression process are suppressed magnificently by the TGUs.

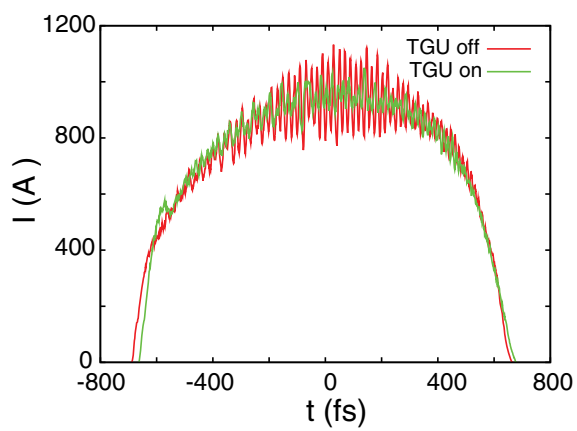

(a) Longitdudinal beam current profile at the exit of TGU2

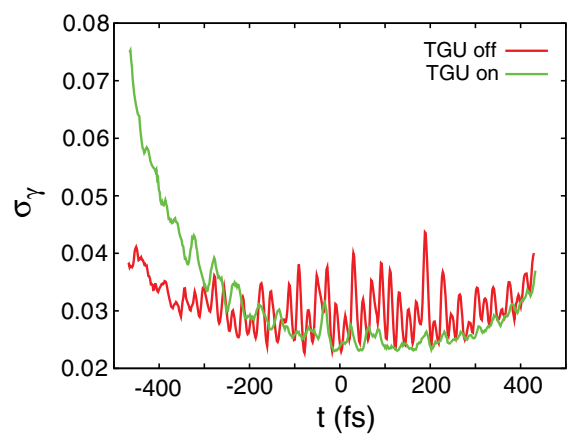

(b) Slice energy profile of the central part of the beam at the exit of TGU2

FIG. 4. Longitudinal beam current and slice energy profile at the exit of TGU2 with TGUs on (green) and off (red).

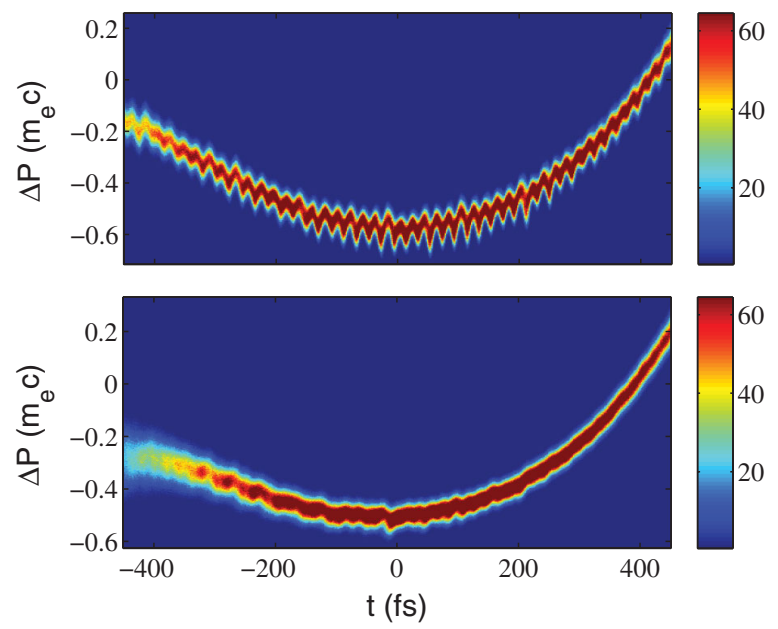

FIG. 5. Longitudinal phase space (time momentum) of the central part of the beam with the energy chirp removed at the exit of TGU2 with TGUs off (upper) and on (lower).

To illustrate the problem, the longitudinal phase spaces of the central part of the beam at the exit of TGU2 with the energy chirp removed are also shown in Fig. 5 with the TGUs on and off, in which one can see that the microstructures are smeared out significantly with the scheme we proposed.

As the result, the simulations above demonstrate that the smooth distribution of the longitudinal phase space can be obtained by the TGUs via the transverse-to-longitudinal phase space coupling. Meanwhile, we can also see that the slice energy spread almost does not change with the TGUs on and off, which indicates that the additional slice energy spread introduced by TGU1 is restored very well by TGU2. For comparison, the additional slice energy spread introduced by a laser heater to suppress the microbunching instability can hardly be recovered after all due to its own physical mechanism [15], which might become a considerable value in some cases. Thus the restoration of the additional slice energy spread in the TGU scheme could be considered as an advantage over a laser heater.

In the last part of this section, it ought to be pointed out that as what is described in Sec. II B, without the second TGU, there will be a significant increase of the horizontal emittance after compression. For the example, if TGU2 is off, the normalized horizontal emittance goes to about $40 \mathrm{~mm} \mathrm{mrad}$ after BC, which is a dramatically large number. To prove the analytical method for preserving the transverse emittance discussed in Sec. II B, first we found a solution of the quadrupole strength $\tilde{k}$ in the linear equations [Eq. (13)] to obtain the matrix form (14) in the linear approximation, then we employed the exactly same value of $\tilde{k}$ in the numerical simulation to see the difference between the horizontal emittances obtained before and after compression. As a result, the normalized horizontal emittance in our example is increased from $0.98 \mathrm{~mm} \mathrm{mrad}$ to $1.07 \mathrm{~mm}$ mrad after compression, and $<10 \%$ difference to 
its original value before compression is mainly introduced by the nonlinear effects such as CSR, LSC, higher order matrix elements, etc. during the compression process, which is not a surprise for general cases.

\section{JITTER ANALYSIS}

Analytical and numerical studies in the previous sections give us confidence in the TGU scheme for suppression of the microbunching instability. In practice, important issues like jitter and tolerances must be taken into account in our scheme to make it successfully performed in a real accelerator. In this section, we will have a discussion about the additional jitter sources in the TGU scheme and the sensitivity of them. In our discussion, higher order effects such as edge focusing, magnetic field imperfection, etc. are not included. On the other hand, the TGU is essentially an insertion device like all the other devices of beam optics, thus our discussion can be based on the method of transport matrix.

The jitter due to the voltage and phase instability in a rf structure always plays a very important role in the jitter analysis for accelerators. As described above, the TGU itself does not need an external rf driving power, therefore there is no rf jitter issue introduced by the extra rf sources in the TGUs. However, the rf jitter due to the accelerating structures such as accelerating tubes and rf linearizer, may bring in the jitters of arrival time, transverse emittance and beam size, etc.

As discussed in Sec. II, matrix elements $M_{14}$ and $M_{24}$ in form (10) representing the transverse dispersion can be made to zero by tweaking the quadrupole strength $\tilde{k}$. As a result, there shall be no jitters of transverse emittance and beam size introduced by the beam energy jitter with perfectly chosen $\tilde{k}$. On the other hand, the matrix element $M_{34}$ in matrix (10) (or generally, $R_{56}$ in a six-dimensional transport matrix) can also bring us the arrival time jitter from the energy instability generated by the jitters of the rf voltage and phase. Again, by looking into the matrix form (10) and comparing the $M_{34}$ element with the one in the transport matrix with the TGUs substituted by drifts, the difference between the two $M_{34}$ $\left(R_{56}\right)$ elements with and without the TGUs writes

$$
\Delta R_{56}=\tau^{2}\left(4 L_{B}+2 L_{12}+L_{23}+4 L_{d}-L_{T} / 3\right),
$$

where $\tau$ is the TGU strength and $L_{d}$ is the drift length before and after the quadrupole shown in Fig. 1, $L_{T}$ is the length of the TGUs, and $4 L_{B}+2 L_{12}+L_{23}$ actually represents the total length of the bunch compressor. The thin lens approximation of quadrupoles is employed to derive Eq. (15), and to be consistent with the common expression, $R_{56}$ is used instead of $M_{34}$ in the equation without losing generality.

In a usual case, the TGU strength is a very small number, e.g., in our computation $\tau \approx 0.05$. Therefore with reasonably chosen $L_{d}$, the difference between the $R_{56}$ with and without the TGUs in the lattice can be insignificant, e.g., $<10 \%$ in our simulation. Moreover, assuming there is the jitter of $\tau$ introduced by the electric power fluctuation that is usually on the order of $10^{-3}$, we have $\Delta \tau \approx 5 \times 10^{-6}$ in our case. This value indicates that the jitter of $\tau$ is too small to bring up the nontrivial deviation to the arrival time. Therefore we conclude that the additional arrival time jitter introduced by the TGUs can be negligible.

\section{SUMMARY AND CONCLUSIONS}

In this paper a simple scheme for suppression of the microbunching instability by TGUs in the LINAC of a FEL facility was proposed and studies were carried out in detail both analytically and numerically. In our discussion, the firstorder transport matrix and the thin lens approximation of quadrupoles were employed which are valid in most of the cases. The theoretical analysis shows that the TGU was able to suppress the instability by two factors: the additional slice energy spread and the longitudinal mixing from the transverse spread without changing the direction of the beam propagation. By applying two TGUs symmetrically before and after the bunch compressor, the microbunching instability can be effectively suppressed by those two factors and the beam quality can also be restored very well after all by carefully choosing the TGU parameters. In the simulation, the typical parameters of a mid-energy electron LINAC was employed to demonstrate the feasibility and the efficiency of the TGU scheme, and the result indicates that the scheme we proposed is able to suppress the microbunching instability significantly with the well-preserved transverse emittance and no notable additional jitter and slice energy spread introduced. Moreover, because the TGU scheme does not need external rf power, laser and chicane systems, etc., it has the advantages over the other schemes in terms of high efficiency, less complexity and better jitter tolerance. As a novel method, the TGU scheme opens for us a new way to improve the performance of the x-ray free-electron laser, and can be a good candidate for the microbunching instability control in addition to a laser heater.

\section{ACKNOWLEDGMENTS}

We wish to acknowledge the help of many colleagues in SINAP such as M. Zhang, D. Gu, B. Liu and D. Wang for the useful discussions on the analytical and simulation results, and many useful suggestions from the experts in the other institutes. This work was partially supported by the Major State Basic Research Development Program of China (2011CB808300) and National Natural Science Foundation of China (NSFC), Grants No. 11275253, No. 11675248, No. 11475250 and No. 11322550.

[1] W. Ackermann et al., Operation of a free-electron laser from the extreme ultraviolet to the water window, Nat. Photonics 1, 336 (2007).

[2] P. Emma et al., First lasing and operation of an ångstromwavelength free-electron laser, Nat. Photonics 4, 641 (2010). 
[3] T. Ishikawa et al., A compact X-ray free-electron laser emitting in the sub-ångström region, Nat. Photonics 6, 540 (2012).

[4] J. Amann et al., Demonstration of self-seeding in a hardX-ray free-electron laser, Nat. Photonics 6, 693 (2012).

[5] E. L. Saldin, E. A. Schneidmiller, and M. V. Yurkov, An analytical description of longitudinal phase space distortions in magnetic bunch compressors, Nucl. Instrum. Methods Phys. Res., Sect. A 483, 516 (2002).

[6] S. Heifets, S. Krinsky, and G. Stupakov, Coherent synchrotron radiation instability in a bunch compressor, Phys. Rev. ST Accel. Beams 5, 064401 (2002).

[7] M. Borland et al., Start-to-end simulation of self-amplified spontaneous emission free electron lasers from the gun through the undulator, Nucl. Instrum. Methods Phys. Res., Sect. A 483, 268 (2002).

[8] E. L. Saldin, E. A. Schneidmiller, and M. V. Yurkov, Klystron instability of a relativistic electron beam in a bunch compressor, Nucl. Instrum. Methods Phys. Res., Sect. A 490, 1 (2002).

[9] Zhirong Huang and Kwang-Je Kim, Formulas for coherent synchrotron radiation microbunching in a bunch compressor chicane, Phys. Rev. ST Accel. Beams 5, 074401 (2002).

[10] C. Behrens, Z. Huang, and D. Xiang, Reversible electron beam heating for suppression of microbunching instabilities at free-electron laser, Phys. Rev. ST Accel. Beams 15, 022802 (2012).

[11] J. Qiang, C. E. Mitchell, and M. Venturini, Suppression of Microbunching Instability Using Bending Magnets in Free-Electron-Laser Linacs, Phys. Rev. Lett. 111, 054801 (2013).

[12] E. L. Saldin, E. A. Schneidmiller, and M. V. Yurkov, Klystron instability of a relativistic electron beam in a bunch compressor, Nucl. Instrum. Methods Phys. Res., Sect. A 528, 355 (2004).

[13] S. Di Mitri, M. Cornacchia, S. Spampinati, and S. Milton, Suppression of microbunching instability with magnetic bunch length compression in a linac-based free electron laser, Phys. Rev. ST Accel. Beams 13, 010702 (2010).

[14] S. Di Mitri and S. Spampinati, Microbunching Instability Suppression via Electron-Magnetic-Phase Mixing, Phys. Rev. Lett. 112, 134802 (2014).

[15] Z. Huang et al., Report No. SLAC-PUB-13854, 2009.

[16] S. Spampinati et al., Laser heater commissioning at an externally seeded free-electron laser, Phys. Rev. ST Accel. Beams 17, 120705 (2014).

[17] Z. Huang, M. Borland, P. Emma, J. Wu, C. Limborg, G. Stupakov, and J. Welch, Suppression of microbunching instability in the linac coherent light source, Phys. Rev. ST Accel. Beams 7, 074401 (2004).

[18] C. Feng, T. Zhang, J. Chen, H. Deng, M. Zhang, X. Wang, B. Liu, T. Lan, D. Wang, and Z. Zhao, Measurement of the average local energy spread of electron beam via coherent harmonic generation, Phys. Rev. ST Accel. Beams 14, 090701 (2011).

[19] T. Smith, J. Madey, L. R. Elias, and D. A. G. Deacon, Reducing the sensitivity of a free-electron laser to electron energy, J. Appl. Phys. 50, 4580 (1979).

[20] H. Deng and C. Feng, Using Off-Resonance Laser Modulation for Beam-Energy-Spread Cooling in Generation of Short-Wavelength Radiation, Phys. Rev. Lett. 111, 084801 (2013).

[21] C. Feng, H. Deng, D. Wang, and Z. Zhao, Phase-merging enhanced harmonic generation free-electron laser, New J. Phys. 16, 043021 (2014).

[22] C. Feng, D. Huang, H. Deng, Q. Gu, and Z. Zhao, Suppression of microbunching instability via a transverse gradient undulator, New J. Phys. 17, 073028 (2015).

[23] M. Cornacchia, S. Di Mitri, G. Penco, and A. A. Zholents, Formation of electron bunches for harmonic cascade X-ray free electron lasers, Phys. Rev. ST Accel. Beams 9, 120701 (2006).

[24] G. Penco, M. Trovo, and S. M. Lidia, Proceedings of FEL2006, THPPH027, Berlin, Germany, 2006 (JACoW, Geneva, 2006).

[25] G. Penco, M. Danailov, and A. Demidovich, E. Allaria, G. De Ninno, S. Di Mitri, W. M. Fawley, E. Ferrari, L. Giannessi, and M. Trovó, Experimental Demonstration of Electron Longitudinal-Phase-Space Linearization by Shaping the Photoinjector Laser Pulse, Phys. Rev. Lett. 112, 044801 (2014).

[26] Z. Huang et al., Measurements of the linac coherent light source laser heater and its impact on the X-ray free-electron laser performance, Phys. Rev. ST Accel. Beams 13, 020703 (2010).

[27] Z. Huang, Y. Ding, and C. B. Schroeder, Compact X-ray Free-Electron Laser from a Laser-Plasma Accelerator Using a Transverse-Gradient Undulator, Phys. Rev. Lett. 109, 204801 (2012).

[28] D. C. Carey, K. L. Brown, and F. Rothacker, Reports No. SLAC-R-530 and No. Fermilab-Pub-98-310, UC-414, 1998.

[29] D.-Z. Huang, Q. Gu, Z. Wang, M. Zhang, and K. Y. Ng, Analysis of the microbunching instability in a mid-energy electron linac, Chin. Phys. C 39, 038101 (2015).

[30] M. Venturini, R. Warnock, and A. Zholents, Vlasov solver for longitudinal dynamics in beam delivery systems for X-ray free electron lasers, Phys. Rev. ST Accel. Beams 10, 054403 (2007).

[31] SXFEL feasibility study report, 2011.

[32] Y. Wang and M. Borland, Pelegant: A Parallel Accelerator Simulation Code for Electron Generation and Tracking, AIP Conf. Proc. 877, 241 (2006).

[33] H. Deng, T. Lin, J. Yan, D. Wang, and Z.-M. Dai, Threedimensional numerical investigations of the laser-beam interactions in an undulator, Chin. Phys. C 35, 308 (2011). 